\title{
Paroxysmal otalgia treated with microvascular decompression of the intermediate nerve: illustrative case
}

\author{
Leonie Witters, MD, ${ }^{1}$ Anton Lukes, MD, PhD, ${ }^{2}$ and Tomas Menovsky, MD, $\mathrm{PhD}^{1}$ \\ ${ }^{1}$ Department of Neurosurgery, Antwerp University Hospital, Edegem, Belgium; and ${ }^{2}$ Department of Neurosurgery, Lindenhofspital, Bern, Switzerland
}

BACKGROUND Intermediate nerve neuralgia is a rare type of cranial neuralgia that causes clinical, therapeutic, and diagnostic challenges. Studies have described pharmacological and surgical treatment options. Surgical treatment ranges from sectioning of neural structures to microvascular decompression. Given the rareness of the disease, there are no clear recommendations concerning treatment.

OBSERVATIONS Reported is the case of a patient with typical intermediate nerve neuralgia. In this particular case, decision-making toward surgical decompression in an earlier stage of the disease could have been beneficial. The authors found excellent results using only microvascular decompression without sectioning of neural structures.

LESSONS Knowledge of intermediate nerve anatomy is essential to understand this complex pain syndrome. This case illustrates that surgery should not only be regarded as a last resort in case of failure of conservative treatment but also should be considered early in the disease course, especially in the case of a clear neurovascular conflict. When no evident cause is found, surgery could be considered as an exploratory option to depict a neurovascular conflict intraoperatively. Microvascular decompression of the intermediate nerve without sectioning of neural structures can obtain excellent results. Since neural structures are saved, postoperative sequelae can be limited.

https://thejns.org/doi/abs/10.3171/CASE2155

KEYWORDS intermediate nerve; intermediate nerve neuralgia; otalgia; microvascular decompression

Intermediate nerve neuralgia (INN), also referred to as "geniculate neuralgia," represents a rare type of cranial neuralgia. According to the International Headache Society, INN is characterized by paroxysmal episodes of pain in the auditory canal with a duration of seconds to minutes in the absence of clinically evident neurological deficits. ${ }^{1}$ The posterior wall of the auditory canal may be a trigger zone. INN can be associated with disorders of lacrimation, salivation, and taste. This chronic pain syndrome is debilitating for patients with INN, but it also poses diagnostic and therapeutic challenges because of the lack of large-scale studies and recommendations given the rareness of the disease.

Reported is a case of a patient with typical INN. In this particular case, decision-making toward surgical decompression in an earlier stage of the disease could have been beneficial. Surgery should therefore be kept in mind as an early treatment option in INN.

\section{Illustrative Case}

We describe a case of a 64-year-old male who experienced intermittent episodes of intractable pain in the auditory canal for 3 years. Three-dimensional constructive interference in steady-state magnetic resonance imaging (MRI) proved neurovascular impingement at the root entry zone (REZ) of the acousticofacial bundle by an elongated vertebral artery (VA) (Fig. 1). First, pain control was attempted pharmacologically with carbamazepine. The patient initially responded well to medication. However, this progressively lost efficiency, and the patient eventually had to be sedated and intubated because of unbearable otalgia.

As a last resort, urgent surgical treatment with microvascular decompression (MVD) of the acousticofacial bundle was considered. Through a retrosigmoid keyhole approach, the intermediate nerve (IN) was mobilized from the offending VA without sectioning. A Teflon paddy was placed between the VA and IN and secured with fibrin glue to prevent recurring impingement. Patency of the VA was confirmed with a micro-Doppler probe. A hemostatic matrix was used for hemostasis. During closure, mastoid air cells and the dura were sealed watertight with the aid of a sealant matrix, and the osseous defect was reconstructed with calcium phosphate.

ABBREVIATIONS CT = computed tomography; IN = intermediate nerve; INN = intermediate nerve neuralgia; MRI = magnetic resonance imaging; $\mathrm{MVD}=$ microvascular decompression; REZ = root entry zone; VA = vertebral artery.

INCLUDE WHEN CITING Published May 10, 2021; DOI: 10.3171/CASE2155.

SUBMITTED January 28, 2021. ACCEPTED February 7, 2021.

(C) 2021 The authors, CC BY-NC-ND 4.0 (http://creativecommons.org/licenses/by-nc-nd/4.0/). 


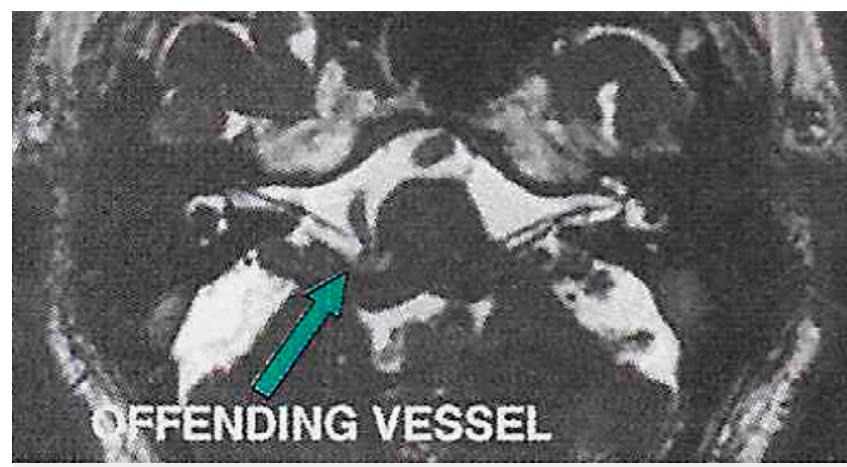

FIG. 1. Three-dimensional constructive interference in steadystate MRI showing the neurovascular conflict at the REZ of the acousticofacial bundle by an elongated VA.

The postoperative course was uneventful. After surgery, the patient noticed immediate pain relief, and administered analgesics were tapered and stopped. The patient's facial, auditory, vestibular, gustatory, and sensitive functions remained normal. A computed tomography (CT) scan confirmed the correct position of the Teflon paddy. The patient was discharged on the seventh postoperative day. He was followed up clinically and remained pain free without medication.

\section{Discussion}

\section{Anatomy}

To understand INN, knowledge of IN anatomy is essential (Fig. 2). The structure's name is derived from the intermediate position between the facial and superior vestibular nerves. During its intracisternal course, the IN adheres medially to the superior portion of the vestibular nerve and then joins the motor root of the facial nerve laterally upon entering the internal acoustic meatus. ${ }^{2}$ The IN is considered to be a part of the facial nerve. The latter has four functional components. ${ }^{3,4}$ The somatic efferent component, originating in the facial motor nucleus in the pons, is responsible for the motor innervation of facial muscles. The IN contains the other three components of the facial nerve: visceral efferent fibers, somatic afferent fibers, and gustatory afferent fibers. The visceral efferents originate in the superior salivary nucleus, consist of preganglionic parasympathetic fibers, and give rise to two branches. The first is the greater petrosal nerve, which synapses in the pterygopalatine ganglion and innervates the main and ancillary lacrimal glands. The second joins the chorda tympani and lingual nerve, synapses in the submandibular ganglion, and innervates the sublingual and submandibular salivary glands. The somatic afferent fibers transmit sensory information from the pinna, the external auditory meatus, and adjacent retromastoid area. They synapse in the geniculate ganglion, which is located in the petrous temporal bone at the level of the genu of the facial nerve, and then follow the motor component of the facial nerve in the opposite direction toward the brainstem. The last component is responsible for taste in the anterior two-thirds of the tongue, the floor of the mouth, and part of the palate. These gustatory afferent fibers run through the lingual nerve and the chorda tympani nerve before synapsing in the geniculate ganglion.

\section{Terminology, Etiology, and Pathophysiology}

INN refers to a chronic pain syndrome of the ear concerning the sensory branch of the facial nerve. The main cause of INN is a neurovascular conflict at the REZ of the IN. This zone, where the transition of myelinating cells from oligodendrocytes centrally to Schwann cells peripherally takes place, shows increased susceptibility to mechanical irritation. The pulsation of vessels can cause

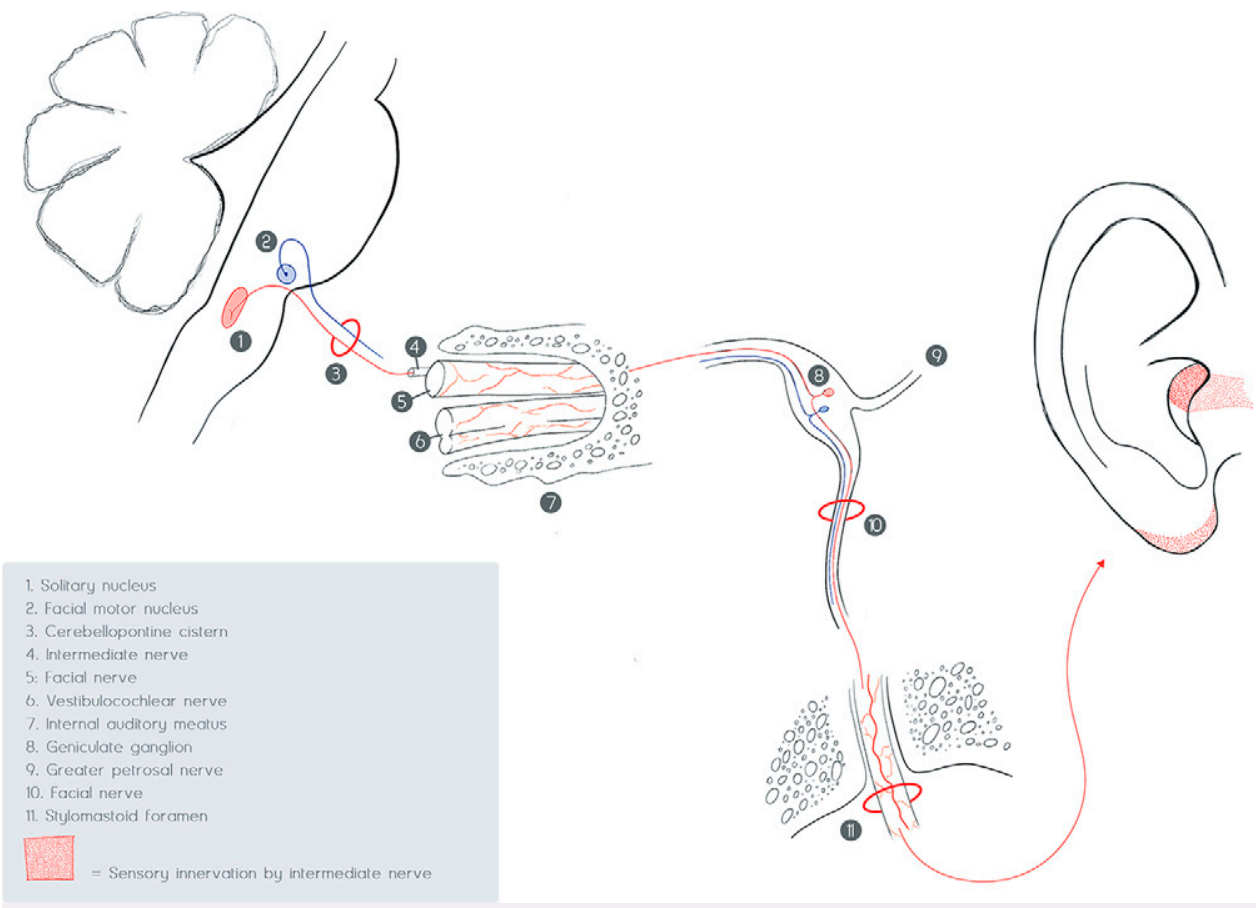

FIG. 2. Anatomical overview of the IN. 
repeated microtraumas, giving rise to focal demyelination followed by aberrant remyelination and the appearance of neoreceptors. This leads to ectopic discharges and ephaptic transmission between neighboring axons. ${ }^{5-7}$ The offending vessel is usually a vascular loop of the anterior inferior cerebellar artery, which is in close anatomical relation with the seventh and eighth cranial nerves. ${ }^{8}$ In addition, atherosclerosis can cause ectatic distortion of vascular loops, causing neurovascular compression. Other case reports have identified the posterior inferior cerebellar artery, the superior cerebellar artery, and the VA as offending vessels. ${ }^{9}$ Furthermore, a venous loop, an aneurysm, and a persistent primitive or hypertrophied acoustic artery can cause INN. ${ }^{10}$

\section{Differential Diagnosis and Diagnosis}

The diagnosis of INN can be challenging. First of all, chronic otalgia has a broad differential diagnosis. ${ }^{9}$ Nonneuralgic causes of otalgia should be ruled out. These causes can be of dental or temporomandibular joint origin or can be situated in the ear. Second, different types of cranial neuralgias can present with otalgia. The sensory innervation of the ear is complex and is provided by multiple nerves: the fifth, seventh, ninth, and tenth cranial nerves but also the second and third cervical nerves. Facial neuralgias that occur most frequently and therefore are the most important differential diagnosis are trigeminal and glossopharyngeal neuralgias. This broad differential diagnosis requires a thorough neurological, dental, and ear, nose, and throat examination. These can also include pure tone audiometry, brainstem evoked response audiometry, vestibular function tests, and a CT scan. High-resolution MRI or magnetic resonance angiography of the cerebellopontine angle is essential, since it might help in confirming compression of the concerning nerve by adjacent vessels and distinguishing INN from other pain syndromes. ${ }^{9,11,12}$ However, this examination is not $100 \%$ sensitive, and it is even less specific. Therefore, when no definite diagnosis is found but clinical suspicion of INN is high, surgery could be considered as an exploratory option to depict a neurovascular conflict intraoperatively.

\section{Treatment}

Different treatment options exist for INN. As in other neuralgias, pharmacological treatment is usually regarded as a first-line treatment. These include antiepileptics such as carbamazepine and gabapentin, tricyclic antidepressants such as amitriptyline, or a combination of these drugs. ${ }^{9}$ Patients with INN may respond well to these medications, especially in early stages of the disease. However, intolerability to side effects and refractoriness of symptoms often occur. ${ }^{13}$

When conservative management fails, invasive procedures are considered. In time, different surgical approaches have been suggested as treatment for INN. ${ }^{9}$ In the past, sectioning of the IN was performed, either intracranially or at the level of the geniculate ganglion. ${ }^{12,14}$ This could be combined with sectioning of other cranial nerves, taking into account the overlapping sensory innervation of the ear. During the past few decades, MVD of the IN at the REZ has been proposed as a treatment option for INN, since the most frequent cause appeared to be a neurovascular conflict in this area. ${ }^{12,14-16}$ When this technique was first applied, MVD was combined with sectioning of the IN. ${ }^{12,14}$ Ever since, discussion has continued regarding whether MVD of the IN alone suffices as treatment or whether it should be combined with sectioning of the IN. Pulec ${ }^{17}$ reported that not only the $\mathrm{IN}$ but also the geniculate ganglion and part of the facial nerve had to be sectioned to achieve satisfying results. A few years later, Lovely and Jannetta ${ }^{14}$ reported that excision of the IN and geniculate ganglion sufficed as an effective treatment. However, sectioning of neural structures gives rise to important and permanent sequelae. For example, sectioning of the IN leads to a decrease of salivation, lacrimation, taste in the anterior two-thirds of the tongue, and sensation in part of the auricle. Also, it can sometimes be difficult to distinguish the IN from the facial and vestibulocochlear nerves, so complications after sectioning can include facial paralysis, hearing loss, and vertigo. Given these complications, MVD alone could be superior to sectioning of the IN. A review of the literature revealed that case reports using only MVD of the $\mathrm{IN}$ are scarce but show good to excellent results. ${ }^{18}$ In this case report, excellent results were found using only MVD of the IN without sectioning of neural structures. Complications of MVD of the IN include neurological deficits such as partial hearing loss or facial nerve palsy due to manipulation of the neural structures. However, these are usually transient side effects. ${ }^{11,19}$

More recently, extracranial intratemporal division of the sensory auricular branch of the facial nerve has been proposed as a less invasive treatment option. ${ }^{20}$ This technique shows promising results, since it is safer than but equally as effective as intracranial procedures, but it still needs validation in larger study groups.

\section{Observations}

Reported is the case of a patient with typical INN. In this particular case, decision-making toward surgical decompression in an earlier stage of the disease could have been beneficial. We found excellent results using only MVD of the IN without sectioning of neural structures.

\section{Lessons}

Knowledge of IN anatomy is essential to understand this complex pain syndrome. This case illustrates that surgery not only should be regarded as a last resort in case of failure of conservative treatment but also should be considered early in the disease course, especially in the case of a clear neurovascular conflict. When no evident cause is found, surgery could be considered as an exploratory option to depict a neurovascular conflict intraoperatively.

MVD of the IN without sectioning of neural structures can obtain excellent results in patients with INN. Since neural structures are saved, postoperative sequelae can be limited in this way.

\section{References}

1. Headache Classification Committee of the International Headache Society (IHS). The International Classification of Headache Disorders, 3rd edition (beta version). Cephalalgia. 2013;33(9):629-808.

2. Rhoton AL Jr, Kobayashi S, Hollinshead WH. Nervus intermedius. J Neurosurg. 1968;29(6):609-618.

3. Guinto G, Guinto Y. Nervus intermedius. World Neurosurg. 2013;79 $(5-6): 653-654$.

4. Hunt J. Geniculate neuralgia (neuralgia of the nervus facialis): a further contribution to the sensory system of the facial nerve and its neuralgic conditions. Arch Neurol Psychiatry. 1937;37(2): 253-285.

5. Love S, Coakham HB. Trigeminal neuralgia: pathology and pathogenesis. Brain. 2001;124(pt 12):2347-2360.

6. Rappaport ZH, Govrin-Lippmann R, Devor M. An electron-microscopic analysis of biopsy samples of the trigeminal root taken during microvascular decompressive surgery. Stereotact Funct Neurosurg. 1997; $68(1-4$ pt 1):182-186. 
7. De Ridder D, Møller A, Verlooy J, et al. Is the root entry/exit zone important in microvascular compression syndromes? Neurosurgery. 2002;51(2):427-434.

8. Yurtseven T, Savaş R, Koçak A, et al. Relationship between anterior inferior cerebellar artery and facial-vestibulocochlear nerve complex: an anatomical and magnetic resonance images correlation study. Minim Invasive Neurosurg. 2004;47(5):306-311.

9. Tang IP, Freeman SR, Kontorinis G, et al. Geniculate neuralgia: a systematic review. J Laryngol Otol. 2014;128(5):394-399.

10. Kempe LG, Smith DR. Trigeminal neuralgia, facial spasm, intermedius and glossopharyngeal neuralgia with persistent carotid basilar anastomosis. J Neurosurg. 1969;31(4):445-451.

11. Sakas DE, Panourias IG, Stranjalis G, et al. Paroxysmal otalgia due to compression of the intermediate nerve: a distinct syndrome of neurovascular conflict confirmed by neuroimaging. Case report. J Neurosurg. 2007;107(6):1228-1230.

12. Rupa V, Saunders RL, Weider DJ. Geniculate neuralgia: the surgical management of primary otalgia. J Neurosurg. 1991;75(4):505-511.

13. De Simone R, Ranieri A, Bilo L, et al. Cranial neuralgias: from physiopathology to pharmacological treatment. Neurol Sci. 2008;29 (suppl 1):S69-S78.

14. Lovely TJ, Jannetta PJ. Surgical management of geniculate neuralgia. Am J Otol. 1997;18(4):512-517.

15. King WA, Wackym PA, Sen C, et al. Adjunctive use of endoscopy during posterior fossa surgery to treat cranial neuropathies. Neurosurgery. 2001;49(1):108-116.

16. Penkert G. Intermedius neuralgia. Article in German. HNO. 1986; 34(9):389-393.

17. Pulec JL. Geniculate neuralgia: long-term results of surgical treatment. Ear Nose Throat J. 2002;81(1):30-33.

18. Goulin Lippi Fernandes E, van Doormaal T, de Ru S, et al. Microvascular decompression of the VII/NIII cranial nerve complex for the treatment of intermediate nerve neuralgia: a retro-spective case series. Oper Neurosurg (Hagerstown). 2018;15(4):378-385.

19. Bellotti C, Medina M, Oliveri G, et al. Neuralgia of the intermediate nerve combined with trigeminal neuralgia: case report. Acta Neurochir (Wien). 1988;91(3-4):142-143.

20. Ulubil SA, Eshraghi AA, Telischi FF. Sectioning the sensory auricular branch of the facial nerve to treat recalcitrant otalgia. Otol Neurotol. 2009;30(4):522-524.

\section{Disclosures}

The authors report no conflict of interest concerning the materials or methods used in this study or the findings specified in this paper.

\section{Author Contributions}

Conception and design: all authors. Acquisition of data: Witters. Analysis and interpretation of data: Witters, Menovsky. Drafting the article: all authors. Critically revising the article: Lukes, Menovsky. Reviewed submitted version of manuscript: Witters, Menovsky. Approved the final version of the manuscript on behalf of all authors: Witters.

\section{Supplemental Information}

Previous Presentations

The abstract was presented as a poster presentation during the Annual Scientific Meeting of the Belgian Society of Neurosurgery, Leuven, Belgium, held on April 10, 2019.

\section{Correspondence}

Leonie Witters: Antwerp University Hospital, Edegem, Belgium. leonie.witters@student.uantwerpen.be. 